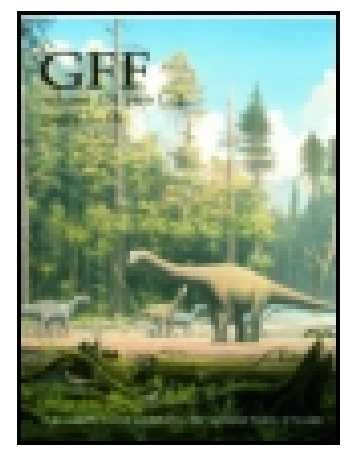

Geologiska Föreningen i Stockholm Förhandlingar

\title{
Le monde scandinave sismique
}

\section{F. de Montessus de Ballore}

To cite this article: F. de Montessus de Ballore (1894) Le monde scandinave sismique, Geologiska Föreningen i Stockholm Förhandlingar, 16:3, 225-230, DOI: 10.1080/11035899409442262

To link to this article: http://dx.doi.org/10.1080/11035899409442262

曲 Published online: 06 Jan 2010.

Submit your article to this journal $\asymp$

Џ Article views: 4

Q View related articles $\sqsubset$ 
GEOL. FürEx, FörhaNDL, N:0 157. Bd 16. .11äft: 3; 225

Le monde scandinare sismique.

Par

F. DF Montessus de BaLlore.

Lidée que les phénoménes sismiques sont d'ordre purement gćologique gagne de plus en plus de terrain, et maintenant il en est peu d'une certaine importance qui ne soient étudiés dans ce sens. Mais en réalité on fait ressortir les influences du terrain, de sa nature, de ses formes et de ses accidents sur la propagation des tremblements de terre, plutôt qu'on ne trouve rien sur leurs causes. C'est pour cela qque ces études particulières, pour. intéressantes qu'elles soient, ne peuvent donner la clef du probléme sismique, encore si obscur et mystéricux.

La première question à résoudre est celle de la connaissance des caractéres aceologiques et autres qui différencient les régions a tremblements de terre de celles dont le sol est plus ou moins stable, et pour $y$ arriver il faut d'abord établir ce que les anglais appelleraient le mgeological survey of the world. Le présent travail a trait aux pays scandinaves.

La nécessité de suivre cette marche est évidente, et la connaissance du globe au point de vue sismique, aussi parfaite que peuvent le permettre les 60,000 séismes connus et enrégistrés sur toute sa surface, fera décourrir quels éléments autres que la nature du terrain interviennent dans la question. Cést qu'en efiet cette nature ne suffit point pour différencier à elle seule les régions à tremblements de terre des autres, car si.l'on range les terrains dans l'ordre du nombre de seismes qui $y$ ont pris nais- 
sance, on ne retrouve point du tout l'ordre dans lequel se présentent les surfaces qu'ils occupent sur le globe -- on devait s'y attendre a priori par ce que les tremblements de terre naissent it une certaine profondeur petite ou grande, peu importe, mais inconnue, où le terrain n'est en général pas le mème qu'ì la surface, là où s'observent les effets du choc. Il s'agit de savoir si le relief relatif du sol, le voisinage des chaines de montagnes ou de la mer, les grandes lignes de corrugation de l'écorce terrestre, la proximité des volcans, les failles, les filons, etca, ont ou non une infuence sur la sismicité des régions, et il semble que tous ces facteurs doivent intervenir, autant sion plus que la nature géologique du terrain superficiel lui-mème.

Ayant ainsi défini le but de ces monographies sismiques, il faut dire comment se mesure la ssismicité, d’une région déterminée par le groupement géographique des points qui ont èté centres de tremblements de terre. On ne peut tenir compte que de la fréquence moyenne plus ou moins bien connue des séismes daus la région consilérée, et non de leur intensité, faute d'un moyen rationnel de mesure de ce dernier élément. Heureusement l'observation montre que li fréquence et l'intensité marchent de pair, et par suite on peut se contenter de la fréquence moyenne.

D'une façon générale si dans une région de surface $S$ on a observé $n$ séismes pendant une période de $p$ années, on appellera sismicité de cette région l'inverse de l'aire de cette région pour láquelle il tremble moyennement une fois par an. En pratique il est plus comnode de prendre cette aire même $\frac{p S}{n}$, de sorte que les régions sont d'autant plus sujettes aux tremblements de terre que le module $\frac{\mathrm{pS}}{\mathrm{n}}$ est plus petit. Naturellement la valeur scientifique ou le poids de ces déterminations varic avec la valeur intrinséque des études sismiques dans chaque pays, et la longueur $p$ des périodes d'observations dont on dispose. Dans chaque cas particulier le lecteur sera averti de la valeur des chiffes donnés. 
GEOL. FÖREX. FöRHANDI. N:0 157. Bd 16. Häft. 3. 227

Un millier environ de tremblements de terre forment la base des détails qui suivent. Les nombres qui accompagnent les noms de localités sur la carte ci-jointe indiquent les nombres de jours que ces lieux ont été centres de tremblements de terre, mais non le nombre de fois, beaucoup plus considérable, qu'ils ont été seconés.

11 tremble relativement peu dans la péninsule scandinave a l'exception d'une région isolée comprenant le littoral norvégien je Bodö à Hemnes, et s'étendant vers l'intérieur jusqu'à Saltdalen. Elle a été étudiée par DAas de 1819 il 1829 . Cette détermination est donc excellente. Isa sismicité est de $1.363 \mathrm{~km}$.

'Toujours sur le littoral on rencontre ensuite le Sündmöre, dont l'axe est le Bremangerfiord. Mais la sismicité est tout de suite très inférieure à celle de la rigion précedente, seulement 16,847 $\mathrm{km}^{2}$. Cette valeur est assez exacte, quoique ne résultant pas d'observations tout-i-fait suivies.

Puis vient l'Islande. Malgré l'importance et la célébrité des éruptions de ses volcans, la sismicité de cette ile, mal connue coutefois faute d'observations suffisantes, n'est certainement pas très grande. Le développenent de ces monographies montre en effet que volcans et tremblements de terre ne vont pas toujours de pair, et que si les éruptions sont fréquemment accompagnées de secousses, ce n'est pas toujours près d'eux qu'il tremble le plus fort et. le plus souvent. La sismicité calculée de l'Islande, mais erronnée par défaut, est de $18,286 \mathrm{~km}^{2}$.

Les coites du golfe de Bothnie viennent ensuite, avec une sismicité assez bien déterminée de $26,273 \mathrm{~km}^{2} ;$ mais on ne sait pas bien jusqu'i quelle distance cette region, qui commence i Söderhaınn, s'étend dans l'intérieur, ni si elle se retourne sur les còtes russes du golfe. En tout cas la Laponie est très rarement secouce; on n’y connait qu'un seul séisme signalé à Kola, dans Ia Laponie russe.

Le reste de la Norvége a une sismicité trés faible de 60,500 $\mathrm{km}^{2}$, car il faut observer que beaucoup de séismes signalés sur ses côtes, en particulier de Bergen à Stavanger, ont leurs centres 
228 F. DE MONTESSUS. LE MONDE. SCANDINATE SISMIQLU.

\section{Carte sismique les Etats Scandinares}

par

F. de Montessus de Ballone.

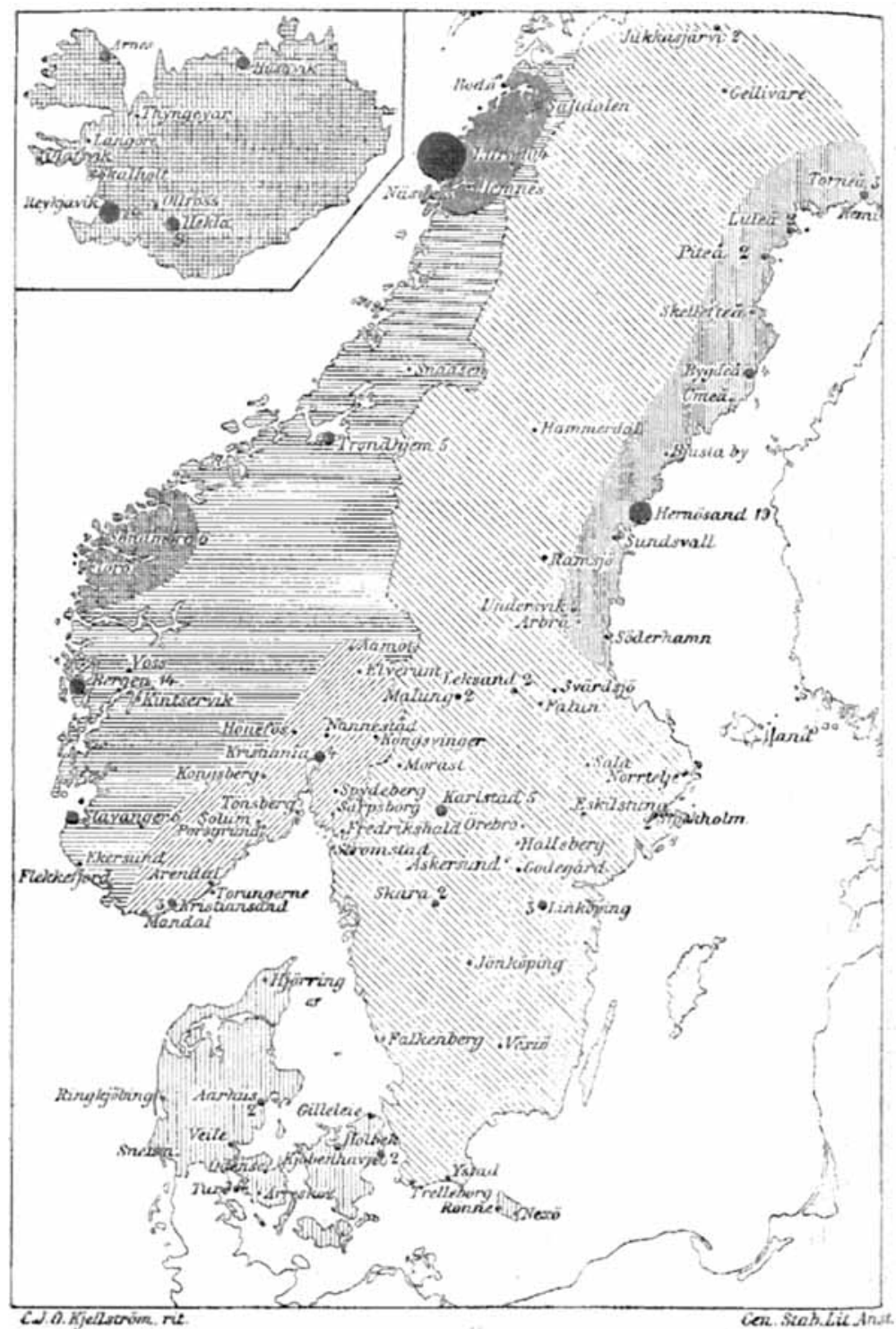


GEOL: FÖREN. FöRHANDL. N:o 157. Bd 16.: Häft. 3. 229

dans les. iles Britanniques ou la mer du nord; et par suite ne doivent pas:lui.être attribués.

Le Jutland et l'archipel danois ont úne sismicité bien déterminée de $68,278 \mathrm{~km}^{2}$. Cette région avoisine l'ammence pays plat qui s'étend du Zuyderzee à la Poméranie et à la.Pologne, ou il ne tremble pour ainsi dire jamais.

Sismicités des direrses régions en kilonétres carrés.

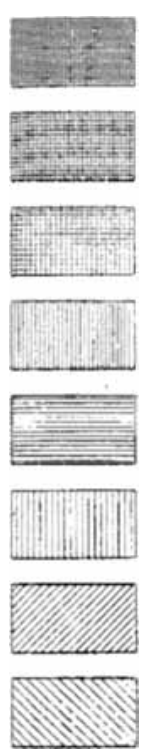

Lurö . • . . . . . . . . . . . . . . $\quad{ }_{1,363}^{k m^{2}}$

Söndmöre . . . . . . . . . . . 16,847

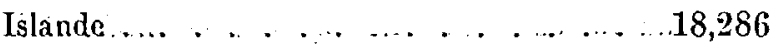

Còtes du golfe de Bothnie.. . . . . 26,273

Reste de la Norvége.... . . . 60,500

Danemark . . . . . . . . . . . 68,278

Christiania . . . . . . . . . 91,854

Reste de la Suéde....... . 221,000

La Norrége présente encore une autre région sismique bien définie géographiquement, celle de Christiania. Elle comprend le littoral de Mandal à Fredrikshall et Ia partie inférieure de Ia vallée du Glommen jusqu'à Aamot; au nord-ouest elle est limitée par les pentes orientales du massif montagneux qui forme la province du Buskerud. Sismicité de $91,854 \mathrm{~km}^{2}$.

Malgré d'assez nombreux centres sismiques dans le gouvernement de Kopparberg, d'Örebro et d'Ostergothland, le reste de la Suéde ne présente qu'une sismicité insignifiante de $221,000 \mathrm{~km}^{2}$. 
Faute de documents il n'a pas été possible de calculer la sismicité du Groenland; elle est certainement très faible; on ny connait que 5 tremblements de terre dont un seul pour la còte orientale, observé par la fameuse expédition de NAxsEx.

On notera qu'aucune conclusion géologique, géographique ou autre n'est donnce ici - c'est à dessein. Il n'y a en effet rien à tirer de l'étude sismique d'une région isolée, mais seulement de l'ensemble.

Belle-1le-en-mer, le 15 decembre 1893.

\section{Errata.}

Il faudra dans la carte ajouter le chiffre 6 à Husavik en Islande et le chiffre 3 à Saltdalen en Norvége. 